\section{Basis for targeting BTK}

\section{By Kai-Jye Lou, Staff Writer}

The CGI Pharmaceuticals Inc. unit of Gilead Sciences Inc. and Roche's Genentech Inc. unit have jointly identified a new inhibitor of the tyrosine kinase BTK and elucidated two BTK-mediated inflammatory pathways involved in arthritis. ${ }^{1}$ The data give mechanistic validation to small molecule BTK inhibitors in clinical development from Avila Therapeutics Inc. and Pharmacyclics Inc. and also describe a distinct mode of inhibition that could be exploited in developing new compounds targeting the kinase.

Bruton's tyrosine kinase (BTK), a member of the Tec family of tyrosine kinases, is involved in both cancer and autoimmune diseases like rheumatoid arthritis (RA). Despite the links to multiple diseases, BTK's biological role is not well understood because a lack of highly selective BTK inhibitors has impeded efforts to tease out the kinase's function.

To address this, Genentech and CGI carried out a screening and SAR study that led to the discovery of CGI1746 (G-182), a potent and highly selective imidazopyrazine-based inhibitor that binds to and stabilizes BTK in an inactive conformation with an $\mathrm{IC}_{50}$ value of $1.9 \mathrm{nM}$. In a panel of Tec and Src family tyrosine kinases, CGI1746 showed more than 1,000-fold selectivity for BTK over other kinases.

The researchers used CGI1746 to show that B cell receptormediated BTK signaling is essential for the production of arthritisassociated autoantibodies. They also showed that BTK is a key mediator of inflammatory cytokine production in response to Fc $\gamma$-receptor (FCGR) signaling (see Figure 1, "BTK-mediated inflammatory pathways in B cells and myeloid cells").

In a mouse model of B cell-dependent arthritis, prophylactic CGI1746 blocked the production of disease-associated autoantibodies and significantly decreased disease severity compared with dexamethasone or vehicle.

In mouse models of myeloid cell-dependent arthritis, CGI1746 prevented the production of inflammatory cytokines and disease development compared with vehicle.

Finally, in mice with established arthritis, CGI1746 significantly decreased disease symptoms compared with vehicle $(p<0.0001)$ and had efficacy comparable to that of blocking tumor necrosis factor- $\alpha$ (TNF- $\alpha$ ).

Results were published in Nature Chemical Biology.

"Unlike previously described Btk inhibitors, CGI1746 exploits binding interactions with an inactive enzyme conformation to deliver over 1,000-fold target selectivity, enabling interrogation of
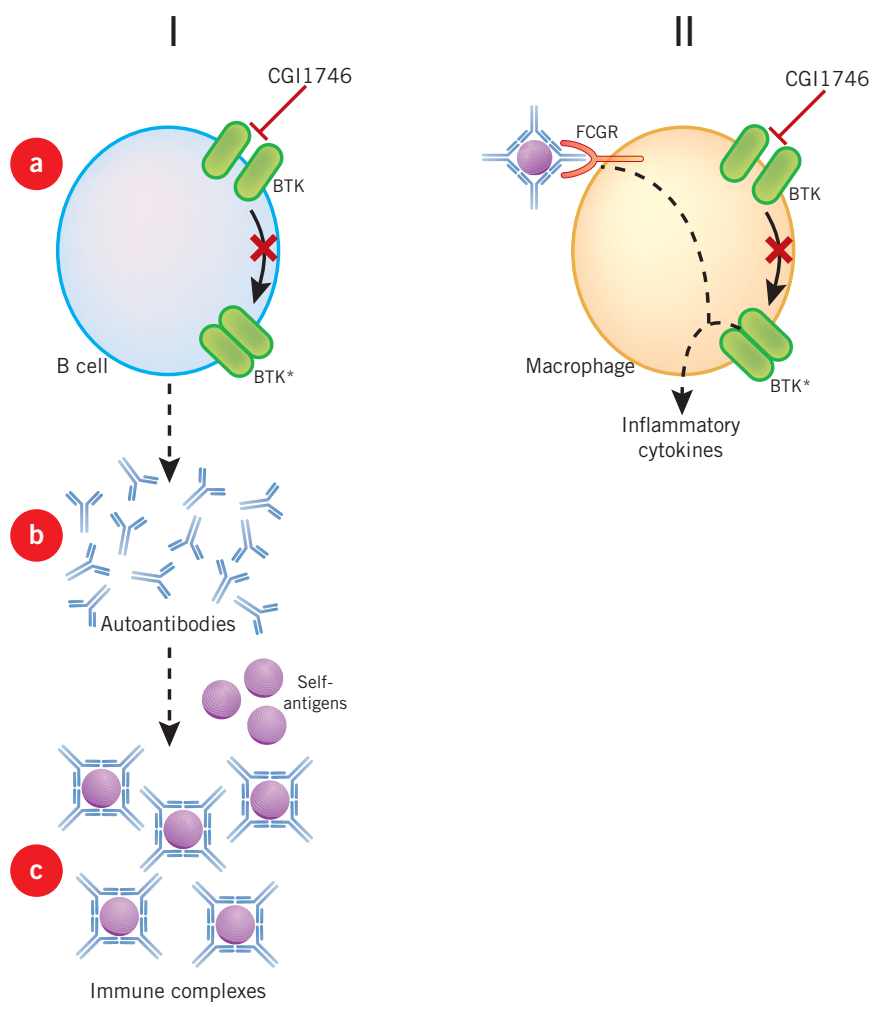

Figure 1. BTK-mediated inflammatory pathways in B cells and myeloid cells. Researchers from the CGI Pharmaceuticals Inc. unit of Gilead Sciences Inc. and Roche's Genentech Inc. unit used CGI1746 (G-182), their newly discovered selective Bruton's tyrosine kinase (BTK) inhibitor, to tease out the cellular and molecular mechanisms behind BTK-mediated inflammation. CGI1746 binds to and stabilizes BTK in an inactive conformation.

The researchers reported in Nature Chemical Biology that CGl1746-mediated BTK inhibition blocks two inflammatory pathways - one mediated by B cells and the other by myeloid cells.

(I) In B cells, CGl1746 blocks B cell receptor-mediated BTK activation $\left(\mathrm{BTK}^{*}\right)$ by preventing the phosphorylation of a key activating tyrosine residue by an upstream kinase [a]. The CGI1746bound, inactive form of BTK is unable to phosphorylate itself and downstream targets. Those downstream targets promote B cell proliferation and the production of arthritis-associated autoantibodies [b] that can form immune complexes with self-antigens [c].

(II) In macrophages and monocytes, which are myeloid lineage cells, immune complexes can trigger the production of inflammatory cytokines through Fc $\gamma$-receptors (FCGRs). The researchers showed that in human monocytes and murine macrophages, BTK is a key mediator of inflammatory cytokine production downstream of FCGRs. In these cells, CGI1746-bound BTK is unable to promote the production of inflammatory cytokines in response to immune complex-mediated FCGR activation. 
Btk function free from the confounding effects of off-target activity," the paper's authors wrote. "Our data highlight a dual mechanism of action of Btk inhibition in inflammatory arthritis, comprising suppression of myeloid cell-dependent inflammatory cytokine production and inhibition of B cell-dependent autoantibody production."

"Most impressive is the high degree of specificity of this inhibitor and the clear demonstration that both B lymphoid and myeloid functions of BTK are inhibited in several animal models without obvious side effects," said Owen Witte, a professor of microbiology, immunology and molecular genetics at the University of California, Los Angeles and an investigator at the Howard Hughes Medical Institute.

Witte, who headed one of the labs that discovered BTK in the 1990s, thinks it is still too early to define how such inhibitors will be used in the RA setting. However, he told SciBX that the chronic use of standard treatments like steroids and TNF blockers have serious side effects. Thus, new compounds with lower toxicities that can be used either as alternatives or in combinations will have to be considered.

"The results in this paper are completely supportive of the data we generated with our own BTK inhibitors," added Juswinder Singh, cofounder and CSO of Avila. "This work will further stimulate interest in BTK as a novel immunomodulatory target."

\section{Looming competition}

Gilead referred questions to Genentech, which declined to comment on the paper; neither company has a disclosed BTK inhibitor in its pipeline.

Gilead's CGI unit holds patents covering imidazopyrazine-based compounds and their use in inhibiting BTK. In 2006, CGI out-licensed its small molecule BTK inhibitors program to Genentech but retained rights in the EU. The partners have since cofiled additional patents covering other classes of compounds and their use as BTK inhibitors.

Meanwhile, Avila and Pharmacyclics both have BTK inhibitors in Phase I trials.

In September, Avila started a Phase I study of AVL-292, a small molecule BTK inhibitor, in healthy volunteers. Data are expected in early 2011. Singh said the company will advance the program in oncology patients next year. "We are also exploring its potential in autoimmune disease in parallel," he added.

Pharmacyclics has two BTK inhibitors in its pipeline: PCI-32765, which is in Phase I testing to treat B cell non-Hodgkin's lymphoma (NHL), and PCI-45292, which is in preclinical development for autoimmune diseases. Earlier this month, the company presented updated Phase I data for PCI-32765 showing a $52 \%$ overall response rate in 48 evaluable patients. The compound was well tolerated. Data were presented at the American Society of Hematology meeting in Orlando, Fla.

Although it is still too early to compare the safety and efficacy of BTK inhibitors, Singh noted that CGI1746 is a reversible BTK inhibitor whereas AVL-292, as well as Pharmacyclics' molecules, are irreversible inhibitors.

"The power of our approach is that we irreversibly inhibit and silence BTK, and thus cells must depend on protein resynthesis to restore BTK signaling," he told SciBX. "We believe the ability of our compound to selectively and covalently bind to BTK to shut down and silence its activity will allow us to achieve complete inhibition against the target, which could translate into strong potency and selectivity."

Pharmacyclics declined to comment.

Lou, K-.J. SciBX 3(48); doi:10.1038/scibx.2010.1431

Published online Dec. 16, 2010

REFERENCES

1. Di Paolo, J.A. et al. Nat. Chem. Biol.; published online Nov. 28, 2010; doi:10.1038/nchembio.481

Contact: Kevin S. Currie, CGI Pharmaceuticals Inc., Branford, Conn. e-mail: kevin.currie@gilead.com

Contact: Karin Reif, Genentech Inc., South San Francisco, Calif. e-mail: kreif@gene.com

COMPANIES AND INSTITUTIONS MENTIONED

Avila Therapeutics Inc., Waltham, Mass.

CGI Pharmaceuticals Inc., Branford, Conn.

Genentech Inc., South San Francisco, Calif.

Gilead Sciences Inc. (NASDAQ:GILD), Foster City, Calif.

Howard Hughes Medical Institute, Chevy Chase, Md.

Pharmacyclics Inc. (NASDAQ:PCYC), Sunnyvale, Calif.

Roche (SIX:ROG; OTCQX:RHHBY), Basel, Switzerland

University of California, Los Angeles, Calif. 